\title{
Hierarchical and Linear Sequence Processing: An Electrophysiological Exploration of Two Different Grammar Types
}

\author{
Jörg Bahlmann, Thomas C. Gunter, and Angela D. Friederici
}

\begin{abstract}
The present study investigated the processing of two types of artificial grammars by means of event-related brain potentials. Two categories of meaningless CV syllables were applied in each grammar type. The two grammars differed with regard to the type of the underlying rule. The finite-state grammar (FSG) followed the rule (AB) ${ }^{n}$, thereby generating local transitions between As and Bs (e.g., $n=2, \mathrm{ABAB}$ ). The phrase structure grammar (PSG) followed the rule $\mathrm{A}^{n} \mathrm{~B}^{n}$, thereby generating center-embedded structures in which the first $\mathrm{A}$ and the last $\mathrm{B}$ embed the middle elements (e.g., $n=2$, $[\mathrm{A}[\mathrm{AB}] \mathrm{B}])$. Two sequence lengths $(n=2, n=4)$ were used.
\end{abstract}

\section{INTRODUCTION}

In a recent article, Fitch and Hauser (2004) raised the question of whether humans and nonhuman primates (i.e., monkeys) differ in their ability to process hierarchical grammatical structures. The authors presented both species with two types of artificial grammar rules. Both humans and nonhuman primates mastered a simple rule with only local transitions between auditorily presented categories of syllables. In contrast, the monkeys failed to master a complex rule with a hierarchical structure of syllable sequences, whereas humans easily processed such structures. The present study addresses the question of which cognitive mechanisms underlie the processing of these two types of artificial grammar rules in humans. Therefore, we examine the processing of a simple, local rule (finite-state grammar [FSG]) and a complex, hierarchical rule (phrase structure grammar $[\mathrm{PSG}]$ ) using the method of event-related brain potentials (ERPs).

Electrophysiological investigations of structural language processes have utilized different types of violations in sentences. Language studies that examined phrase structure violations have reported an early left anterior negativity (ELAN), which was interpreted as a

Max Planck Institute for Human Cognitive and Brain Sciences, Germany
Violations of the structures were introduced at different positions of the syllable sequences. Early violations were situated at the beginning of a sequence, and late violations were placed at the end of a sequence. A posteriorly distributed early negativity elicited by violations was present only in FSG. This effect was interpreted as the possible reflection of a violated local expectancy. Moreover, both grammar-type violations elicited a late positivity. This positivity varied as a function of the violation position in PSG, but not in FSG. These findings suggest that the late positivity could reflect difficulty of integration in PSG sequences.

reflection of an initial phrase structure building process, and a late positivity called the P600, representing syntactic reanalysis or repair (Hahne \& Friederici, 1999, 2002; Friederici, Pfeifer, \& Hahne, 1993; Neville, Nicol, Barss, Forster, \& Garrett, 1991). Morphosyntactic agreement violations have been associated with a left anterior negativity (LAN) and a late positivity (Osterhout \& Nicol, 1999; Coulson, King, \& Kutas, 1998; Münte, Heinze, Matzke, Wieringa, \& Johannes, 1998; Gunter, Stowe, \& Mulder, 1997; Friederici et al., 1993). For instance, Münte et al. (1998) tested semantic, morphosyntactic, and orthographic violations in German sentences. Whereas semantic violations correlated with a classical N400 plus P600 pattern, morphosyntactic violations caused an early negativity and a late positivity. The early negativity was interpreted as a detection mechanism in response to the morphosyntactic error, and the late positivity was hypothesized to reflect a syntactic reanalysis process. Semantic, morphosyntactic, and syntactic violations were also used in the experimental design by Friederici et al. (1993). An N400 component was elicited by semantic violations, whereas morphosyntactic violations elicited a negativity and a late positivity, and syntactic violations elicited an ELAN.

In contrast to the studies with phrase structure violations or agreement violations, other experiments have varied the dependencies between phrases in a sentence (Fiebach, Schlesewsky, \& Friederici, 2001, 2002; Kaan, 
Harris, Gibson, \& Holcomb, 2000; Rösler, Pechmann, Streb, Röder, \& Hennighausen, 1998; King \& Kutas, 1995; Kluender \& Kutas, 1993; King \& Just, 1991). For example, Kaan et al. (2000) found a late positivity with a higher amplitude for a lexical element in a complex syntactic structure in comparison to a less complex syntactic structure. The complexity of the syntactic structure was also manipulated in a study by Fiebach et al. (2001). In this study, subject- versus object-related wh-questions (e.g., who) were used. A persistent left frontal negativity and a late positivity were reported for the more complex object-related sentences only. The late positivity related to the variation of the long distance dependencies could reflect difficulties in integrating the more complex syntactic structure. A LAN was also found in English sentences with wh-questions in comparison to yes/no questions (Kluender \& Kutas, 1993). Rösler et al. (1998) observed a LAN for German noncanonical sentences when compared to canonical sentences. In both cases, the authors interpreted their findings to reflect the additional load imposed on working memory during the processing of long distance dependencies in sentences.

Only a few ERP experiments exist that have examined the computation of different types of rule-based structures. Recent studies have investigated language-related artificial grammar rules (Friederici, Steinhauer, \& Pfeifer, 2002), syllable sequences with transformational rules (Hoen \& Dominey, 2000), and arithmetical sequences (Nunez-Pena \& Honrubia-Serrano, 2004). Hoen and Dominey (2000) compared transformations in letter sequences with a memory control condition. The transformations followed the rule 123-312 (e.g., ABC-CAB), whereas the memory condition involved recognizing the repetition of a target element at the end of the sequence (e.g., ABC $\underline{\mathbf{Z} D E F} \underline{\mathbf{Z}}$ ). A LAN and a later positivity were found for transformations. The authors suggested that the LAN component represents an intersection of general structure building processes. Nunez-Pena and Honrubia-Serrano (2004) conducted an experiment with numerical series and varied the violation of the series in terms of error salience. An early negativity and a late positivity for the processing of violations were reported. Moreover, the amplitude of the positivity varied as a function of salience: The more salient the error in the sequence, the larger the positivity. This saliency effect was interpreted to reflect different degrees of difficulty in integrating the violated arithmetical structure.

Furthermore, the computation of visually presented sentences and rule-based sequences of letter strings was compared in a study by Lelekov-Boissard and Dominey (2002). In the language (sentence) condition, a new word was inserted in a phrase to create a word category violation in a sentence. In the sequencing (letter string) condition, the letter strings followed a simple transformation rule (e.g., A-B-C B-C-B), and violations of this rule were produced by inserting a new element at the end of the sequence (e.g., A-B-C B-C-므). Sentences and letter string sequences elicited a late positivity for violations, but the effects differed with regard to scalp distribution for the two sequence types. The authors proposed related and overlapping neurophysiological processes for structure building in speech and nonspeech processes. Friederici et al. (2002) used a language-like artificial grammar task to investigate rule processing, in contrast to the simple transformational rules in the studies by Lelekov-Boissard and Dominey (2002) and Hoen and Dominey (2000). This artificial grammar featured pseudowords in different word classes and syntactical rules. The language-related rules were first learned by participants until a certain performance level was reached, then participants were tested with correct and violated sentences. Violations of these structures revealed an early negativity and a late positivity. Overall, the studies discussed above suggest that the processing of both transformational rules and language-like artificial grammar rules show similarities to native language processing.

The serial reaction time task (SRT; Nissen \& Bullemer, 1987) represents a different paradigm in the investigation of structure building and sequencing. In this task, the assessment of sequence learning is analyzed. Visual stimuli appear at different locations on a screen, and response buttons spatially correspond to these locations. The sequences of the stimuli follow a complex, continuously recurring rule. Typically, reaction times decrease over time significantly more for rule-based sequences than for random orders. Some ERP studies have used variations of an SRT, resulting in somewhat conflicting results. Whereas Rüsseler and Rösler (2000) and Eimer, Goschke, Schlaghecken, and Sturmer (1996) found an enhancement of the N200 and P300 component for violations of the regular sequence, Baldwin and Kutas (1997) only found a higher amplitude of the P300 for grammatically structured orders of the letters. In addition, the influence of explicit versus implicit learning of the task on the P300 is still questionable. Both Rüsseler, Hennighausen, Münte, and Rösler (2003) and Eimer et al. (1996) found the N200 and P300 effects only for explicit learners, whereas Baldwin and Kutas (1997) reported no learning-type effect whatsoever. Therefore, the degree to which the processing of the SRT and the processing of natural language use overlapping cognitive mechanisms remains an open question. In this respect, it is interesting to note that patient studies have demonstrated that aphasic and agrammatic patients master the SRT. In contrast, they fail to learn transformations of the rule (Dominey, Hoen, Blanc, \& Lelekov-Boissard, 2003), and they are impaired in learning auditorily presented rule-based phoneme sequences (Goschke, Friederici, Kotz, \& van Kampen, 2001). For example, agrammatic patients showed impairments in a transformation task in which the order of the letter sequence had to be shifted (Dominey et al., 2003). Thus, if the 
N200 and P300 components are assumed by Hoen and Dominey (2000) to be language independent and if aphasic and agrammatic patients successfully perform the SRT, then this suggests different neural generators for the type of sequencing required in the SRT and language-related sequencing.

\section{The Present Study}

The present study was designed to compare violations in locally organized rule-based sequences with violations in hierarchically organized rule-based sequences. According to Chomsky and Miller (1958), an FSG is specified as probabilistic transitions between a finite set of elements. These transitions feature local organizational principles. For instance, the rule $(\mathrm{AB})^{n}$ (e.g., $n=2, \mathrm{ABAB}$ ) generates local transitions between the categories A and $\mathrm{B}$. In contrast, PSGs feature hierarchical structures, allowing for the generation of long-distance dependencies (Chomsky, 1965). A simple example of a PSG is the rule $\mathrm{A}^{n} \mathrm{~B}^{n}$ in which the first $\mathrm{A}$ and the last $\mathrm{B}$ embed the middle elements (e.g., $n=2[\mathrm{~A}[\mathrm{AB}] \mathrm{B}]$ ). Such artificially generated embeddings could be compared to natural sentences such as The boy [that the girl saw] was tall. It is worthwhile to note that in natural speech, the generation of long-distance dependencies is not only provided by the relation between word categories. Other features such as animacy, case, case in combination with gender, verb argument structure, and so on, also provide mechanisms for the interpretation of long-distance dependencies. Unlike in most studies with natural sentences, in this study, only the aspect of category was explored, whereas other features were held constant.

In the present experiment, syllable sequences following the FSG were compared with syllable sequences following the PSG (see Figure 1). In response to violations of both grammatical rule types, the prediction was a late positivity similar to the $\mathrm{P} 600$ component for violations in natural language tasks. Two types of sequence lengths were applied: short sequences $(n=2)$ and long sequences $(n=4)$. The violations of the syllable sequence structures were situated at different

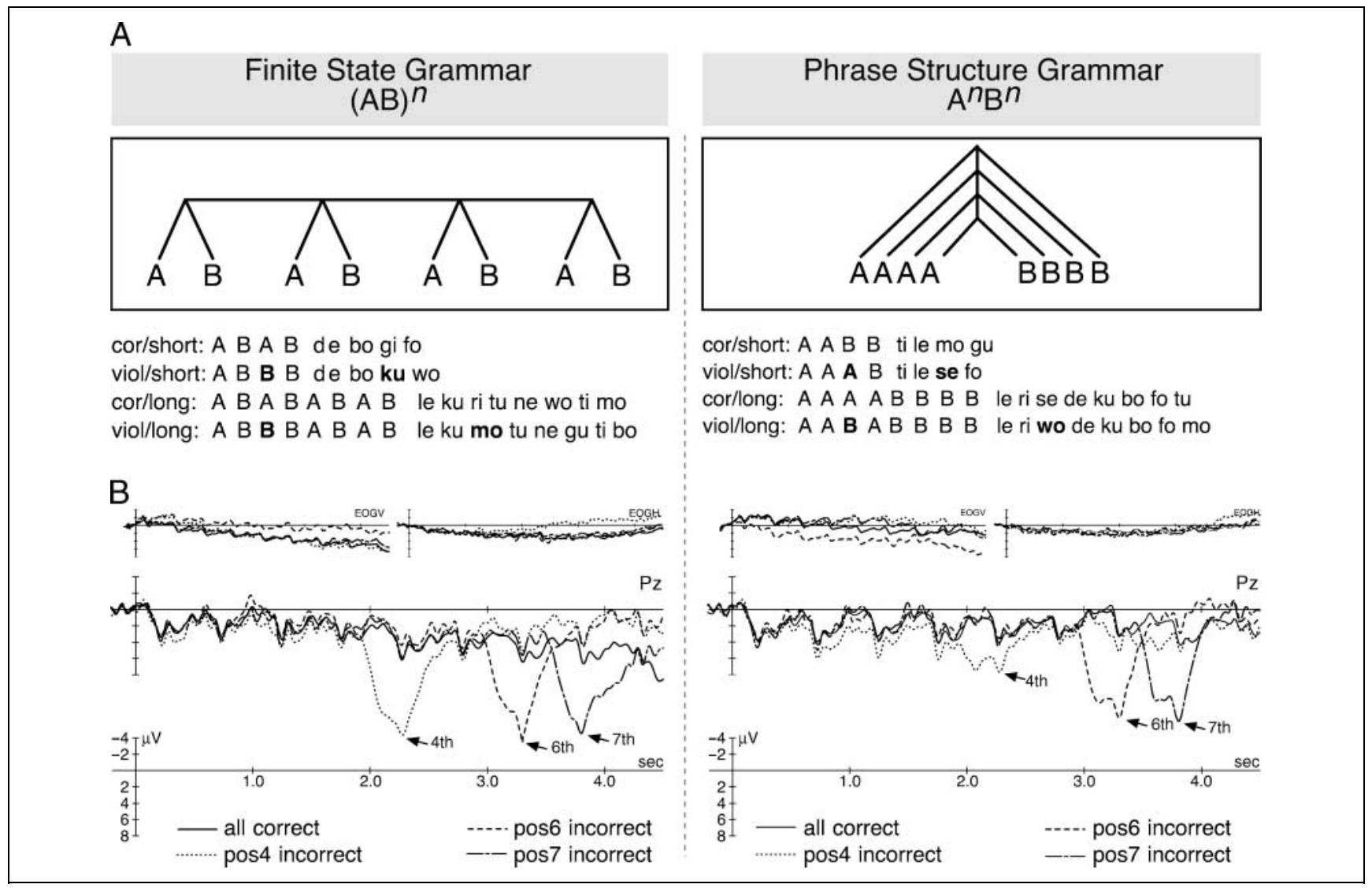

Figure 1. (A) General structure and examples of the stimuli in the FSG and the PSG. The linear structure of the FSG was generated by simple transitions between the two categories of syllables (A and B). The hierarchical structure of the PSG was produced by embeddings between the two syllable categories. Short and long sequences were applied. Violations of the structure were situated at different positions in a sequence for both rule types (FSG and PSG). In the example, the violations are placed at the third position for FSG and PSG and for short and long sequences (bold letters). (B) Example of the grand average ERPs for long sequences from 24 participants. Negative voltage is always plotted upwards. One selected electrode is displayed for FSG (left) and for PSG (right). ERPs of the whole sequence for a correct sequence (all correct, solid line), a sequence with a violation at the fourth position (posu incorrect, dotted line), a violation at the sixth position (pos6 incorrect, dashed line), and a violation at the seventh position (pos7 incorrect, dotted and dashed line). 
positions in the sequences. The violation positions were counterbalanced over the structure types (FSG and PSG) and sequence lengths (short and long). The manipulation of the violation positions ensured that participants needed to focus on the whole syllable sequence structure and not just part of it to complete the error detection task required in the experiment. In addition, this variation made it possible to investigate the ERP correlates at different points in time while processing the different structures. This manipulation allows us to compare violations in the first half of the sequence (early violations) to violations in the second half (late violations). With regard to the respective rule types, it was hypothesized that FSG and PSG would elicit differences in the late positivity for early and late violations. The processing of violations at different positions in a local structure (FSG) should cause no differences in the ERP components. In contrast, violations at an early position in a hierarchical structure (PSG) should elicit a different late positivity than do violations at late positions. A violation at the first position of a sequence should not cause differences in the ERPs for FSG and PSG.

\section{METHODS \\ Participants}

Twenty-four healthy, right-handed subjects participated in this study (12 women, mean age 23 years, $S D=2.4$ ). All participants were native German speakers and had normal or corrected-to-normal vision. All participants gave their written informed consent prior to testing. The data were handled confidentially.

\section{Stimuli}

According to the experiment by Fitch and Hauser (2004), the FSG was coded as (AB) ${ }^{n}$, whereas the PSG followed the rule $\mathrm{A}^{n} \mathrm{~B}^{n}$. Sequences of $\mathrm{CV}$ syllables were visually presented to the subjects. Syllables were assigned to two categories. In the study by Fitch and Hauser, the two categories were distinguished by pitch ( $\mathrm{A}=$ high pitch, $\mathrm{B}=$ low pitch). Due to the visual presentation in the present study, the two categories were coded phonologically. Category A syllables contained the vowels $i$ or $e$ (e.g., de, gi, le, ri, se, ne, $t i, m i)$, and Category $\mathrm{B}$ syllables were composed of $o$ or $u$ vowels (e.g., bo, fo, ku, mo, pu, wo, tu, gu). Examples for syllable sequences are given in Figure 1. For both types of artificial grammar, the same syllables were used. To prevent pattern learning, the probability of occurrence of the frequency of the syllables was balanced in the material. If one syllable occurred by chance more frequently in a certain position of one sequence, then the participant could assume a rule behind this chunk. Hence, all syllables appeared with equal frequency in the experiment. (The last two sentences repeat the same info as in the previous two.)

\section{Procedure}

The experiment was divided into two sessions. One session contained the learning and testing of the FSG, and in the other session, the PSG was trained and tested. Each experimental session contained a learning period followed immediately by a testing period. The sessions were separated by $7( \pm 1)$ days. Due to technical reasons, two participants had a shorter delay between the experimental sessions ( 3 and 4 days) and two participants had a longer delay (13 and 14 days). However, no differences in behavioral data were found for these four participants compared to the others.

\section{Learning}

The learning period consisted of several blocks. At the beginning of each block, 10 correct sequences were presented. Afterward, five correct and five incorrect sequences were shown for which subjects were required to respond with a button press whether the sequences were grammatical or ungrammatical. For each sequence, feedback was given. Participants were instructed to extract the rule underlying the syllable sequences. Training ended when subjects answered 90\% of the items correctly in two successive blocks. For the learning period, 504 syllable sequences were computed. Sequences of four, six, and eight syllables were used (168 items each sequence length).

\section{Testing}

During the testing period, the actual ERP measurement was conducted. Participants were seated in a dimly lit, sound-attenuated chamber facing a computer screen. Participants were instructed to judge whether the sequences were rule-based or not. As in the training, feedback was given. For the testing period, 700 new sequences were presented, that is, 350 short sequences (four syllables) and 350 long sequences (eight syllables) were computed, and half of them (175 for each length) were violated. The start of a sequence was indicated by a fixation cross (1000 msec). Each syllable was presented for 300 msec with an interstimulus interval of $200 \mathrm{msec}$ between the syllables. At this point, participants could make their judgment for $1000 \mathrm{msec}$, which was followed by feedback for $500 \mathrm{msec}$. The trial length was set to $4500 \mathrm{msec}$ (four syllables per sequence), $5500 \mathrm{msec}$ (six syllables), or $6500 \mathrm{msec}$ (eight syllables).

Key assignment, sex of the subjects, and order of the grammar type (FSG first vs. PSG first) was counterbalanced across subjects. To prevent subjects from focusing on one particular position within a sequence during the 
detection of a violation, the position of the violation was systematically changed.

\section{ERP Recording}

The EEG activity was recorded with $56 \mathrm{Ag} / \mathrm{AgCl}$ electrodes mounted in an elastic cap. Electrode impedance was kept below $5 \mathrm{k} \Omega$. Bipolar horizontal and vertical electrooculograms were recorded for artifact-rejection purposes. All scalp electrodes were referenced to the left mastoid. The signals were recorded continuously with a band-pass filter from DC to $30 \mathrm{~Hz}$ at a sampling rate of $250 \mathrm{~Hz}$ and stored on hard disc for off-line analysis.

\section{Data Analysis}

Average ERPs of correct and incorrect syllables were computed for Positions 1, 3, 4, 6, and 7 in both the FSG and PSG. Each average started at $200 \mathrm{msec}$ prestimulus and lasted $1000 \mathrm{msec}$ after the presentation of a syllable and was computed for each electrode position. Only correctly judged sequences entered the analysis. Approximately 3\% of the trials had an ocular artifact. Thus, approximately $6 \%$ of all trials were excluded from further analyses. To avoid a loss of statistical power when repeated measures analyses of variance (ANOVAs) are used to quantify large numbers of electrodes (Oken \& Chiappa, 1986), 10 regions of interest (ROIs) were computed out of the 56 electrodes, each containing the mean of 3 electrodes. Statistical analyses were then performed using five anterior and five posterior ROIs in two latency windows (negativity, 300$400 \mathrm{msec}$; late positivity, 400-750 msec). The analyses differed in the positions of violations within a sequence (see below). Main effects were interpreted at a significance level of $p<.05$. The Greenhouse-Geisser correction (Greenhouse, 1959) was always applied when evaluating effects with more than one degree of freedom in the numerator. In such cases, the uncorrected degrees of freedom $(d f)$, the corrected $p$ values, and the correction factor $\varepsilon$ are reported. For all significant effects, the partial effect size measure $\omega^{2}$ was calculated. The partial effect size reflects the amount of variance of the dependent variable that is explained by the independent variable (Hays, 1973) and ranges between 0 and 1 .

\section{RESULTS \\ Behavioral Data}

The PSG learning took longer than the FSG learning did: PSG, $15.45 \mathrm{~min}$; FSG, $8.25 \mathrm{~min} ; t(23)=3.073, p<.01$. No transformation effects of the order of the learning of the two grammar types, $t(22)=.79, n s$; sex, $t(22)=$ $1.19, n s$; or key assignment, $t(22)=1.62, n s$ were found.
To verify differences in the processing of short and long sequences of FSG and PSG, an ANOVA with the factors grammar type and sequence length (both two-level, within subject) was conducted for error rates. The processing of the PSG resulted in slightly more errors (4\%) than processing of the FSG $(2 \%)$, indicated by a main effect of grammar type: $F(1,23)=4.95, p<.05$, $\omega^{2}=.06$. On the basis of the significant interaction between grammar type and sequence length, $F(1,23)=$ $6.93, p<.05, \omega^{2}=.11$, separate $t$ tests for paired samples were conducted for both grammar types. Error rates for long sequences were significantly higher than for short sequences, $t(23)=2.58, p<.05$ in PSG (short, $3 \%$; long, 5\%), but not in FSG (short, 3\%; long, 2\%), $t(23)=1.16, n s$.

\section{ERP Data}

\section{Long Sequences in FSG and PSG}

To explore the differences between both rule types, the violation positions were compared in detail. In both rule types, the violations elicited a posterior negativity around $350 \mathrm{msec}$ poststimulus followed by a large positivity with its maximum around $700 \mathrm{msec}$. The pattern of the positivity clearly differs between the two types of grammatical rules (see Figure $1 \mathrm{~B}$ and Figure 2). The amplitude of the positivity in the FSG seems to remain equal, independently from the position of the violation within a sequence. Violation at Position 4 seems to have the same positivity effect as violations at the late Positions 6 and 7. In contrast, the position of the violation in a PSG sequence appears to have an influence on the amplitude of the positivity. An error of the rule on an early position (4) elicits a smaller positivity than on a late position ( 6 and 7 ). Due to the temporal ambiguity of violations at Position 3 in the PSG, a direct comparison of FSG and PSG at this position would be inadequate. Consequently, each rule type was separately analyzed. First, a distinct analysis in the FSG condition was calculated to show that the position of violation within a sequence had no influence on the ERP pattern. An ANOVA with the factors position (four), violation (two), anterior/posterior (two), and ROI (five) was conducted. For the factor position, the positions included were 3, 4, 6, and 7. In the PSG condition, Positions 4, 6, and 7 were analyzed to demonstrate that the ERP pattern systematically changed over the violation positions in a sequence. In this analysis, Positions 4, 6, and 7 formed the factor position, and an ANOVA with the factors position (three), violation (two), anterior/posterior (two), and ROI (five) was carried out. The third position did not enter the analysis in the PSG condition because this is an ambiguous position. Thus, the third position was analyzed separately (see below). In both analyses (FSG and PSG) the first position was excluded from the factor position because no differences of the 


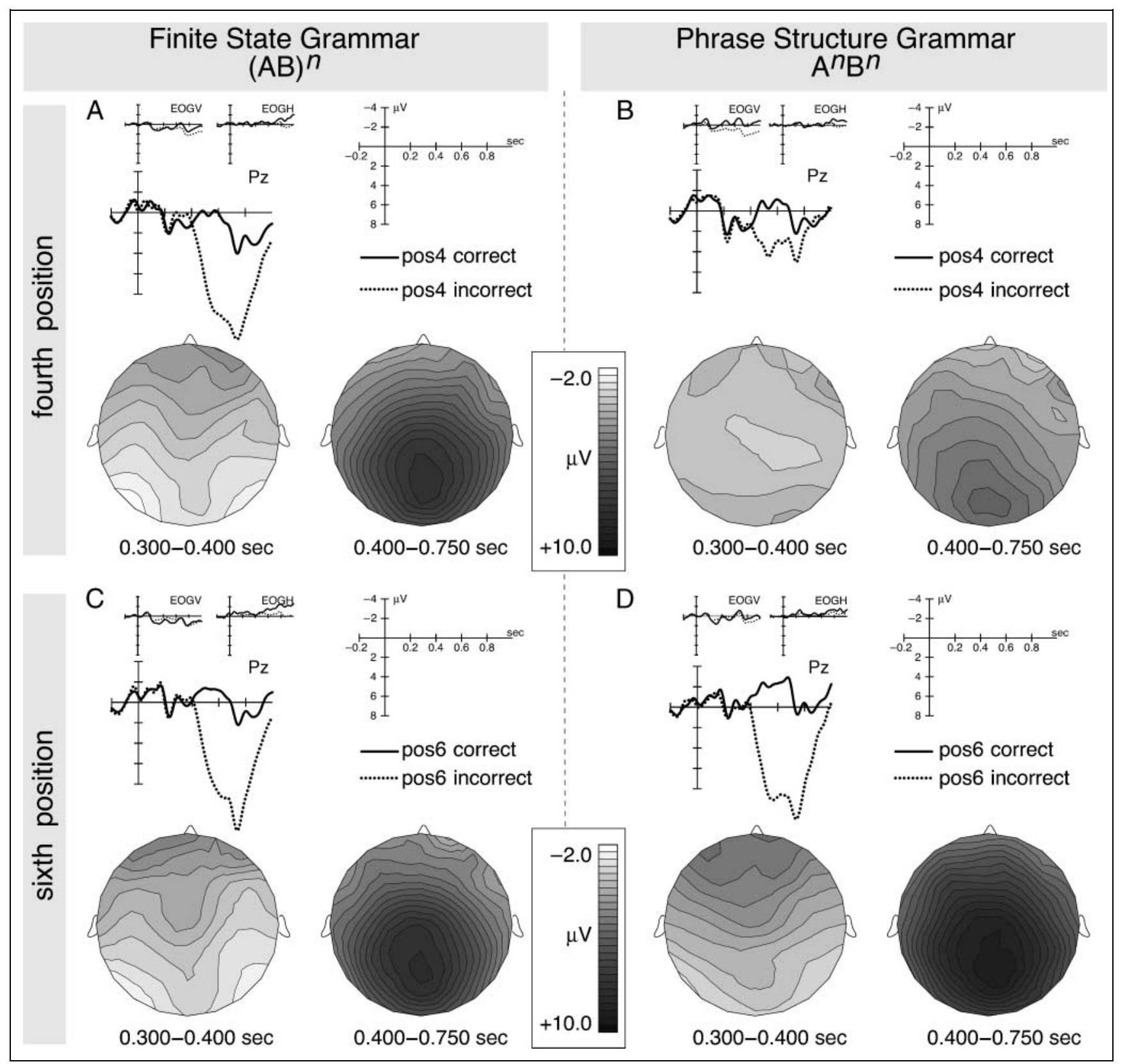

Figure 2. Top: ERPs elicited by FSG (A) and PSG (B) at the fourth position for correct (pos4 correct, solid line) and for incorrect (pos4 incorrect, dotted line) sequences as shown by a selected electrode. Topographic maps of the differences between effects for correct and incorrect sequences at the fourth position are shown for the two time windows that entered analyses. Dark areas indicate positive differences between the conditions, and light areas indicate negative differences. Bottom: ERPs for the sixth position in FSG (C) and PSG (D) for one selected electrode. ERPs of the sixth position for correct (pos6 correct, solid line) and for incorrect (pos6 incorrect, dotted line) sequences are shown. Topographic maps of the ERPs of correct minus incorrect sequences at the sixth position are shown for the two time windows that were entered into the analyses.

ERP pattern of the first position of the two rule types were expected. Hence, the first position was analyzed separately (see below).

FSG: Positions 3, 4, 6, and 7. 300-400 MSEC: POSTERIOR NEGATIVITY. The main analysis of the 300- to 400-msec interval showed no two-way, three-way, or four-way interactions with the factors position and violation (all
$F<2.65, n s)$. A two-way interaction of anterior/posterior and violation, $F(1,23)=24.69, p<.0001, \omega^{2}=.32$, was observed. Inspection of the ERPs showed that this interaction was caused by the posterior scalp distribution of the negativity (Figure $2 \mathrm{~A}$ and $\mathrm{C}$ ). These results indicate that the negativity in FSG is independent of the violation position in a sequence. The negativity does not differ between violations in the first half and the second half of a FSG sequence. 
400-750 MSEC: LATE POSITIVITY. The main analysis for the 400to 750-msec interval showed no two-way, three-way, or four-way interactions with the factors position and violation (all $F<2.75, n s$ ). Significant interactions between anterior/posterior and violation, $F(1,23)=14.86$, $p<.001, \omega^{2}=.21$, and between ROI and violation, $F(4,92)=25.01, \varepsilon=.48, p<.0001, \omega^{2}=.49$, were found. Visual inspection of the ERPs indicates that these interactions were caused by a centroparietal scalp distribution of the late positivity (see Figure 2A and C). This analysis shows the late positivity in the FSG condition is found regardless of violation position. Violations in the first and second half of a FSG sequence show a similar late positivity in the ERPs.

PSG: Positions 4, 6, and 7. 300-400 MSEC: POSTERIOR NEGATIVITY. The main analysis of the 300- to 400-msec interval showed a significant three-way interaction of position, anterior/posterior, and violation, $F(2,46)=15.82, \varepsilon=$ $.82, p<.0001, \omega^{2}=.32$. On the basis of this interaction, separate analyses with the factors anterior/posterior and violation were performed for each position in the PSG condition:

Position 4: No main effect or interaction reached significance at the fourth position (all $F<.92$ ).

Position 6: An interaction of anterior/posterior and violation was found, $F(1,23)=19.06, p<.0005, \omega^{2}=$ .27 . On the basis of this interaction, separate analyses were conducted for anterior and posterior regions. At posterior ROIs, the main effect of violation, $F(1,23)=$ $1.82, n s$, was not significant, confirming that no posterior distributed negativity was found for violations of Position 6 of the PSG. At anterior ROIs, the main effect of violation reached significance, $F(1,23)=$ 12.8, $p<.005, \omega^{2}=.19$. Visual inspection of the ERPs revealed that the main effect of violation at this late position was caused by the earlier onset of the late positivity, earlier than for the positivity at an earlier position (i.e., Position 4; see Figure 2D).

Position 7: A significant three-way interaction between anterior/posterior, ROI, and violation, $F(4,92)=3.44$, $\varepsilon=.60, p<.05, \omega^{2}=.08$, was observed. On the basis of this interaction, separate analyses were carried out for anterior and posterior ROIs at Position 7. In anterior ROIs, an interaction between ROI and violation was observed, $F(4,92)=3.18, \varepsilon=.51, p<.05, \omega^{2}=$ .08 . Based on this interaction, separate analyses were conducted for each of the five ROIs. The two left ROIs and the rightmost ROI showed a significant main effect of violation $(5.5 \leq F \leq 14.44, .001 \leq p \leq .05, .08 \leq$ $\left.\omega^{2} \leq .21\right)$. The main effect of violation did not reach significance in the middle and the middle right ROIs (all $F<2.11, n s$ ). Inspections of the scalp distributions showed a similar pattern as in the anterior ROIs of Position 6: Violations at the late positions (6 and
7) show an earlier onset of the late positivity than violations at Position 4.

400-750 MSEC: LATE POSITIVITY. The main analysis for the 400- to 750-msec interval showed a significant threeway interaction between position, ROI, and violation, $\left.F(8,184)=7.29, \varepsilon=.52, p<.0001, \omega^{2}=.33\right)$. On the basis of this interaction, separate analyses with the factors ROI and violation were performed for each position.

Position 4: A significant main effect of violation was found, $F(1,23)=11.04, p<.005, \omega^{2}=.17$. Furthermore, an interaction between ROI and position was observed, $\left.F(4,94)=4.35, \varepsilon=.51, p<.05, \omega^{2}=.11\right)$. Inspection of the ERPs indicates that this interaction was produced by a centroparietal scalp distribution of the late positivity (see Figure 2B).

Position 6: A significant main effect of violation was obtained, $F(1,23)=97.2, p<.0001, \omega^{2}=.67$. A threeway interaction between anterior/posterior, ROI, and violation was also significant, $F(4,92)=6.05, \varepsilon=.59$, $p<.005, \omega^{2}=.17$. On the basis of this interaction, separate analyses in the five anterior and five posterior ROIs were performed. All five anterior ROIs showed significant main effects of violation $(26.0 \leq$ $F \leq 49.5$, all $\left.p<.0001, .34 \leq \omega^{2} \leq .50\right)$ as did all five posterior ROIs (100.79 $\leq F \leq 114.4$, all $p<$ $\left..0001, .67 \leq \omega^{2} \leq .70\right)$. The larger effect at posterior ROIs $\left(.67 \leq \omega^{2} \leq .70\right)$ in comparison to anterior ROIs $\left(.34 \leq \omega^{2} \leq .50\right)$ assumed a posteriorly distributed late positivity on violations at the sixth position. Inspections of the ERPs showed a broadly distributed late positivity with its peak on the posterior electrodes for violations on the sixth position (see Figure 2D).

Position 7: A significant main effect of violation was elicited, $F(1,23)=125.7, p<.0001, \omega^{2}=.72$. On the basis of a significant three-way interaction between anterior/posterior, ROI, and violation, $F(4,92)=5.42$, $\varepsilon=.56, p<.001, \omega^{2}=.14$, separate analyses in the five anterior and five posterior ROIs were conducted. All anterior and posterior ROIs showed significant main effects of violation (anterior: $31.77 \leq F \leq 55.4$, all $p<.0001, .38 \leq \omega^{2} \leq .53$; posterior: $83.9 \leq$ $F \leq 153.8$, all $\left.p<.0001,63 \leq \omega^{2} \leq .76\right)$. Posterior ROIs showed a larger violation effect $\left(.63 \leq \omega^{2} \leq .76\right)$ than anterior ROIs $\left(.38 \leq \omega^{2} \leq .53\right)$; hence, it was assumed that the late positivity showed a posteriorly distributed late positivity. Inspections of the ERPs showed a broadly distributed late positivity with its peak on the posterior electrodes for violations at the seventh position.

Taken together, violations on the sixth and seventh positions show similar scalp distributions and effect sizes in the PSG condition. In comparison with the two 
positions later in a sequence, violations on the fourth position revealed a different scalp distribution and effect size. Positions 6 and 7 showed a broadly distributed late positivity, whereas Position 4 displayed a posteriorparietal distributed late positivity. The size of the violation main effect on Position $4\left(\omega^{2}=.17\right)$ is smaller than that for Positions 6 and $7\left(\omega^{2}=.67\right.$ and $\left.\omega^{2}=.72\right)$.

To summarize, the effect of violations that were introduced at an early position in the sequences were compared with violations occurring at later positions. This comparison was done in FSG on Positions 3 and 4 (early violations) and on Positions 6 and 7 (late (violations). Due to the temporal ambiguity at the third position in PSG, this position did not enter the analyses of early and late violations in the PSG condition. Hence, Position 4 (early violation) was compared with Positions 6 and 7 (late violations). In the FSG condition, violations at Positions 3, 4, 6, and 7 show a similar ERP pattern. Independent of the position of the violation within a sequence, a negativity followed by a late positivity was elicited. In contrast, in the PSG condition, the ERP pattern changes as a function of the violation position in a sequence.

\section{Short versus Long Sequences in FSG and PSG}

FSG: Positions 3 and 4 in short and long sequences. To verify whether short and long sequences differ in the
ERPs in FSG, the third and fourth position of short and long sequences were compared. An ANOVA with the factors anterior/posterior (two), ROI (five), short/long (two), and violation (two) was conducted. The main analysis for the 300- to 400-msec interval showed no main effect of short/long, $F(1,23)=0.11, n s$, and no interaction with the factor short/long (all $F<.32, n s$ ). In addition, the ANOVA on the 400- to 750-msec interval showed no main effect of short/long, $F(1,23)=0.57$, ns, and no short/long interactions (all $F<1.3, n s$ ). These analyses demonstrate that there is no difference in the ERPs for short and long sequences in FSG.

PSG: Position 3 in short and long sequences as a case of ambiguity. POSITION OF THE VIOLATION IN PSG: THIRD POSITION. In the PSG, a violation at the third position of the sequence should cause ambiguity because a Category A syllable at this position could be a correct long sequence or an incorrect short sequence. Upon visual inspection of the ERPs, there seems to be no difference between incorrect, short sequences (AAAB) and correct, long sequences (AAAABBBB) at the third position. Apparently, also correct, short (AABB) and incorrect, long sequences (AABABBBB) show no differences in the ERPs (see Figure 3). Therefore, it was assumed that participants treat this ambiguous position as if it were a long sequence. If this post hoc hypothesis is true, then

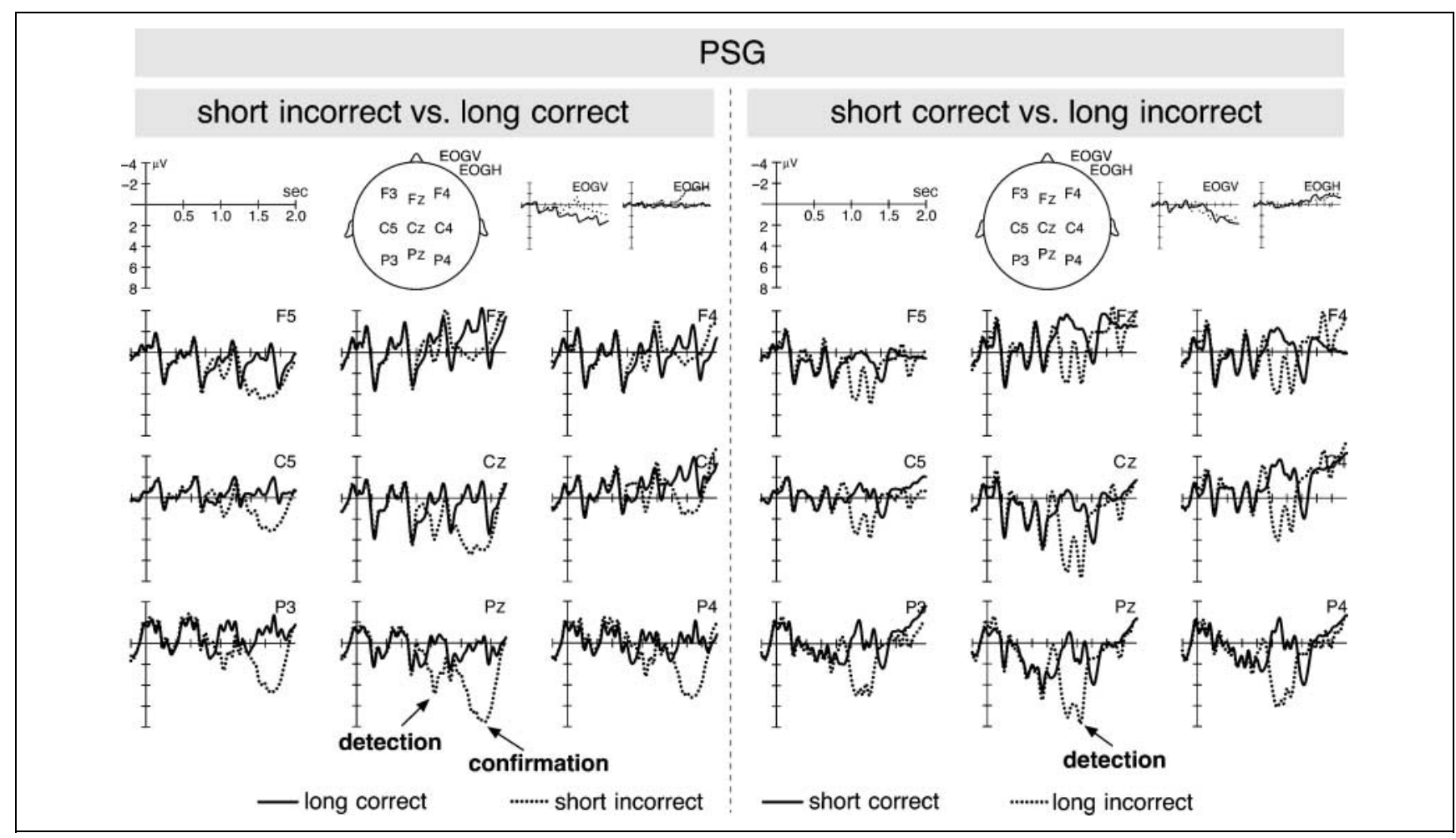

Figure 3. ERPs for the ambiguous third position in the PSG are illustrated. Left: ERPs for short incorrect (AAAB, dotted line) and long correct (AAAABBBB, solid line) sequences are shown at the third position. Right: ERPs for short correct (AABBB, solid line) and long incorrect

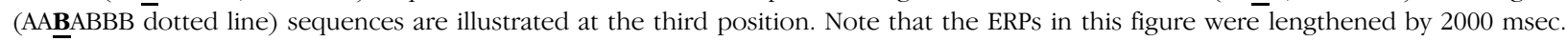


the ERPs found in the incorrect short versus correct long (AAAB vs. AAAABBBB) and correct short versus incorrect long (AA $\underline{\mathbf{B}} \mathrm{B}$ vs. $\mathrm{AA} \underline{\mathbf{B}} \mathrm{ABBBB})$ should show no difference. To test this, two separate analyses were carried out, one with the factors anterior/posterior (two), ROI (five), and incorrect short/correct long (two), and the second ANOVA with correct short/incorrect long (two) as the third factor.

Incorrect short sequences. The analysis for the 300- to 400-msec interval for the first analysis showed no main effect of incorrect short/correct long, $F(1,23)=$ $0.01, n s$, and no interactions with this factor. Also, the analysis for the 400- to 750-msec interval revealed no main effect of incorrect short/correct long, $F(1,23)=$ $0.02, n s$, and no interaction effect with this factor, meaning that the ERPs for correct short and incorrect long sequences at the third position in the PSG are not different.

Incorrect long sequences. In the second analysis at the 300- to 400-msec interval, there was no main effect of correct short/incorrect long, $F(1,23)=0.67$, $n s$, but an interaction of correct short/incorrect long with anterior/posterior, $F(1,23)=4.44, p<.05, \omega^{2}=$ .06. The step-down analysis revealed no main effect of correct short/incorrect long in the anterior ROIs, $F(1,23)=3.24$, ns, and in the posterior ROIs, $F(1,23)=0.2$, ns. The analysis for the 400- to 750-msec interval revealed no main effect of correct short/incorrect long, $F(1,23)=0.03, n s$, and no interactions with this factor. Together, the ERPs for incorrect short and correct long sequences and for correct short and incorrect long sequences at the third position in the PSG do not differ. The above analyses therefore support the assumption that participants dealt with the ambiguous third position by processing it as a correct long sequence.

POSITION OF DETECTION OF THE VIOLATION: FOURTH POSITION. The previous analyses showed that subjects process information at the ambiguous third position as belonging to the long sequence. Although the system managed to deal with this ambiguous position, when does the detection of a violation for a short PSG takes place? In order to analyze the position of violation detection, the ERPs of the third position were lengthened by $2000 \mathrm{msec}$, thereby providing information about when the violation was actually detected. Inspection of the ERPs revealed two different positive components elicited from violations for short and long sequences (see Figure 3). The first positivity occurred at 950 to $1250 \mathrm{msec}$, and the second positivity appeared from about 1450 to $1850 \mathrm{msec}$ after the onset of the third item. To explore the significance of these components, the same type of analyses as in the previous subsection (Position of the violation in PSG: third position) were conducted in the time windows 950-1250 and 14501850 msec.

Incorrect short sequences. An ANOVA with the factors anterior/posterior (two), ROI (five), and incorrect short/correct long (two) was conducted.

The main analysis for the 950- to 1250-msec interval showed an interaction between anterior/posterior and incorrect short/correct long, $F(1,23)=7.2, p<.05, \omega^{2}=$ .11. On the basis of this interaction, separate analyses in anterior and posterior ROIs were carried out. The main effect of incorrect short/correct long was only significant in posterior ROIs, $F(1,23)=13.97, p<.001, \omega^{2}=.20$, but not in anterior ROIs, $F(1,23)=.39$, ns. This effect revealed that in short sequences, the positivity showed a posterior scalp distribution.

The main analysis for the 1450 - to $1850-\mathrm{msec}$ interval also displayed a significant interaction of anterior/posterior with incorrect short/correct long, $F(1,23)=8.59$, $p<.01, \omega^{2}=.13$, leading to the breakdown analysis in anterior and posterior ROIs. In anterior ROIs, the main effect of incorrect short/correct long was not significant, $F(1,23)=4.01$, ns. In posterior ROIs, this effect was significant, $F(1,23)=54.55, p<.0001, \omega^{2}=.52$. Thus, the second positivity showed a posterior distribution and a higher effect size than the first positivity on short sequences.

Incorrect long sequences. An ANOVA with the factors anterior/posterior (two), ROI (five), and correct short/ incorrect long (two) was conducted.

The main analysis for the 950- to 1250-msec interval showed a main effect of correct short/incorrect long, $F(1,23)=39.14, p<.0001, \omega^{2}=.44$. No other effect was significant. Inspection of the ERPs indicates a broadly distributed first negativity for long sequences.

The main analysis for the 1450- to 1850-msec interval showed a three-way interaction between anterior/posterior, ROI, and correct short/incorrect long, $F(4,92)=$ $6.89, \varepsilon=.58, p<.001, \omega^{2}=.17$. On the basis of this interaction, separate analyses were carried out in each of the five anterior and each of the five posterior ROIs. No main effect of correct short/incorrect long reached significance $(.5 \leq F \leq 1.62, n s)$. This analysis indicated that the second positivity was not significant for long sequences.

Taken together, it was shown that the detection of an error that occurred at the third position took place at the fourth position, independent of the length of the sequence. This effect was substantiated by a positivity at 950 to $1250 \mathrm{msec}$ after onset of the third item. Moreover, a second positivity at about 1450 to $1850 \mathrm{msec}$ was elicited by short sequences, but not by long sequences. 


\section{First Position in FSG and PSG}

To explore whether differences between the rule types already occur at the first position of the sequences, an ANOVA with the factors rule type (two), violation (two), anterior/posterior (two), and ROI (five) was carried out for the first position of the sequences.

The main analysis for the 300- to 400-msec interval showed a main effect of violation, $F(1,23)=20.6$, $p<.0001, \omega^{2}=.28$. The interaction between rule type and violation was not significant, $F(1,23)=0.00, n s$. The main analysis for the 400- to 750-msec interval showed a main effect of violation, $F(1,23)=7.41, p<.001, \omega^{2}=$ .11. No other interactions were significant. Taken together, no differences of the ERPs at the first position were found for FSG and PSG (see Figure 4).

\section{DISCUSSION}

In the present study, two types of artificial grammar rules were compared in which two types of categories (A and B) of meaningless syllables were used. The sequences of syllables followed either a locally organized FSG rule or a hierarchically organized PSG rule. The number of syllables per category (eight syllables in each category) and the length of the syllable sequences (four syllables in short and eight syllables in long sequences) were held constant between the two types of grammar rules. Furthermore, due to the within-subject design, the interindividual variance was held constant. The behavioral data suggest that no transfer effect was found for the learning of the grammar rules. Violations of the rules were introduced at different positions of the sequences. A negativity at about 300 to $400 \mathrm{msec}$ was elicited only in FSG and a positivity between 400 to $750 \mathrm{msec}$ was found with different amplitudes for FSG and PSG.

\section{The Late Positivity}

The late positivity for violations of structured sequences are in line with several studies on the processing of language (Hahne \& Friederici, 1999, 2002; Osterhout \& Nicol, 1999; Coulson et al., 1998; Münte et al., 1998; Gunter et al., 1997, 2000; Friederici et al., 1993), languagelike artificial grammar (Friederici et al., 2002), syllable sequences with transformational rules (Lelekov-Boissard \& Dominey, 2002; Hoen \& Dominey, 2000), arithmetical sequences (Nunes-Pena \& Honrubia-Serrano, 2004), and harmonic violations in music (Patel, Gibson, Ratner, Besson, \& Holcomb, 1998). In the experiment by Friederici et al. (2002), a late positivity was observed for category violations in a complex artificial grammar with phrase structure. Hoen and Dominey (2000) reported similar results for nonlinguistic abstract structures; that

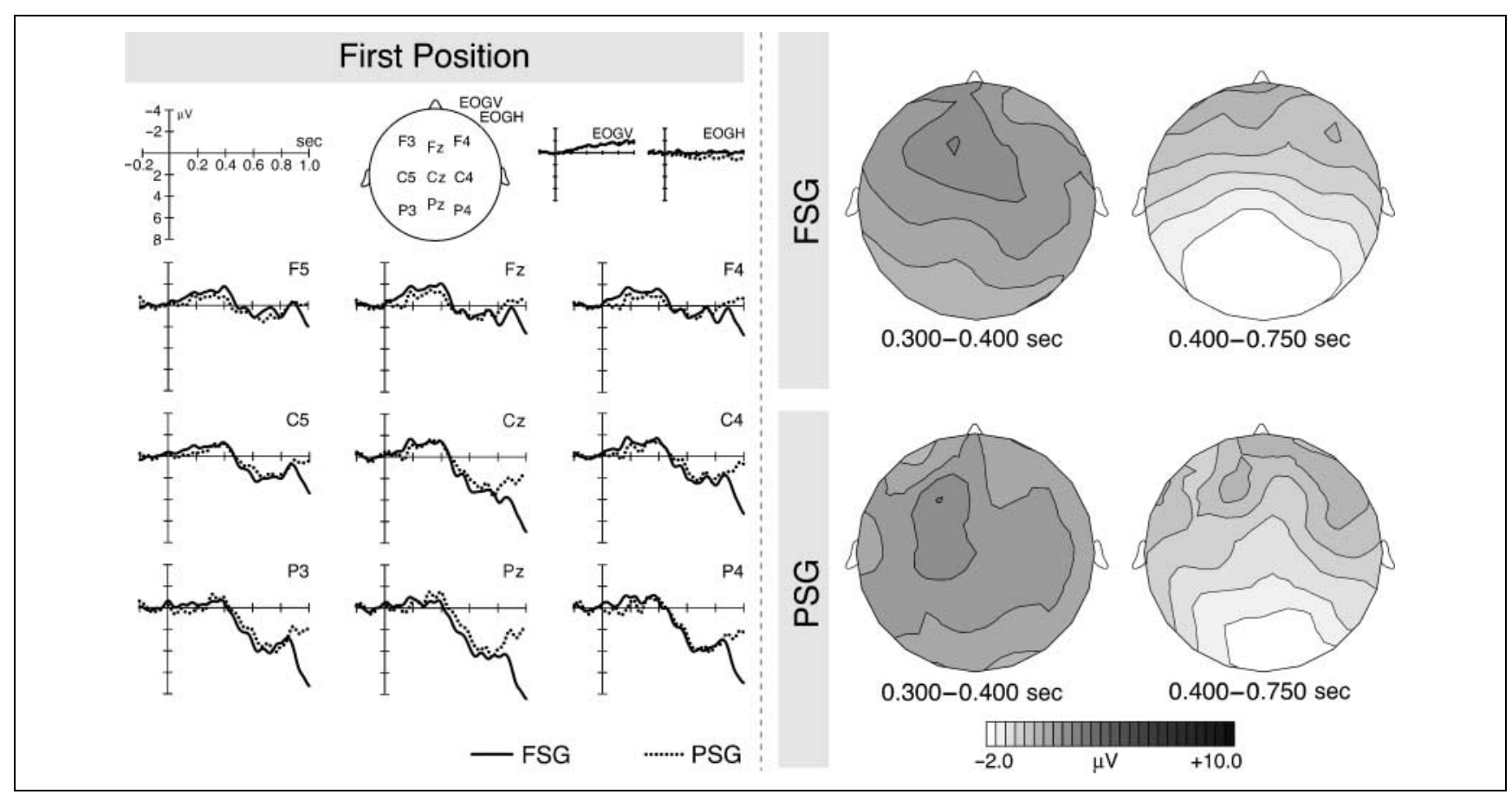

Figure 4. Left: Difference waves of grand average ERPs from 24 participants for nine selected electrodes. Correct and violated sequences of the first position in a sequence in both rule types are shown. Solid lines represent the difference waves of the ERPs from correct minus violated sequences of the FSG, and dotted lines represent the difference waves of the ERPs from correct minus violated sequences of the PSG. Right: Topographic maps of the differences between the ERPs of correct and incorrect sequences of the first position in the sequences. Two time windows are shown for both rule types (FSG in the upper right and PSG in the lower right). Dark areas indicate positive differences between the conditions, and bright areas indicate negative differences. 
is, a late positivity was elicited for transformations in letter sequences. Lelekov-Boissard and Dominey (2002) compared syntactic errors in sentences with errors in a transformation structure, whereas Patel et al. (1998) compared syntactic anomalies in sentences with harmonic violations in music. Although both experiments by Lelekov-Boissard and Dominey and Patel et al. used completely different stimuli, they both report a late positivity evoked by anomalies of the structure. Studies using natural language that have reported a late positivity manipulated the phrase structure by introducing a word category violation (Hahne \& Friederici, 1999, 2002; Friederici et al., 1993) or the morphosyntactic structure (Osterhout \& Nicol, 1999; Coulson et al., 1998; Münte et al., 1998; Gunter et al., 1997, 2000; Friederici et al., 1993). The similarity of the late positivity in the present study to those found in natural language studies suggests at least partly overlapping neuronal generators of structure building in natural language and in an artificial grammar.

\section{The Negativity}

Another interesting finding obtained in the present experiment is that the processing of the FSG elicited a posteriorly distributed negativity at about 300-400 msec. A similar negativity was also reported when an additional item violated the structure of a language-like artificial grammar (Friederici et al., 2002). Eimer et al. (1996) reported a larger negativity for deviant stimuli than for the standard stimuli in an SRT for explicit learners, but not for implicit learners. Rüsseler et al. (2003) found a broadly distributed negativity with a centroparietal maximum for deviants in an SRT paradigm. Again, this was shown only for a participant group that was explicitly aware of the rule. The authors suggested that the conscious recognition of the unfulfilled expectation of a specific item could be reflected by the negativity. This effect could also account for the early posterior negativity in the FSG in the present study. The processing of the local probabilities between the syllable categories lead to an expectation of a specific category; that is, an A is always followed by a B. If this category did not appear, the expectation was not fulfilled and the violation was detected. The posteriorly distributed early negativity in the FSG could reflect this unfulfilled expectation of the specific B category during the processing of the syllable sequences. In addition, violations at the first syllable position (B instead of A) elicited a broadly distributed early negativity and a centroparietally distributed late positivity for both FSG and PSG. This activation pattern can be taken to support the hypothesis that an unfulfilled expectation of a category caused the early negativity, as both rule types correctly start with an A category. The finding that violations of PSG elicited no negativity (except for violations at the first position of a sequence) suggest that the processing of the dependencies in the PSG might require more complex mechanisms than the simple matching of transitional probabilities of syllable categories.

\section{Effects of Violation Position: Early versus Late}

Violations of the syllable sequence structures were introduced at different positions in the sequences. Early violations were located in the first half of the sequence at Positions 3 and 4, whereas late violations occurred in the second half at Positions 6 and 7. The late positivity varied as a function of the structure type. The FSG revealed no differences in the amplitude of the positivity at varying violation positions. In contrast, the PSG elicited different amplitudes of the positivity for early versus late violations within a sequence, with the later positions demonstrating a larger amplitude than the early one.

The FSG is specified as probabilistic transitions between a finite set of elements (Chomsky \& Miller, 1958). In the present case, the local transition remains the same independent of the position. This is reflected in the ERPs. The early negativity and the late positivity in the FSG do not differ in the amplitude or scalp distribution for early and late violations of the structure. In the PSG, however, the position of the violation matters functionally. This is also reflected in the ERPs: There were differences in the positivity size when comparing early and late violation positions. That is, late positions elicited a larger positivity, but we assume that this was not merely an expectancy effect.

In terms of the traditional approach of context updating, variation of the P300 amplitude points toward variations of subjective probability or of modifications in task relevance (Donchin \& Coles, 1988). Subjective probability (Johnson \& Donchin, 1982) or expectancy (Squires, Wickens, Squires, \& Donchin, 1976) was shown to be reciprocally proportional to the P300 amplitude, that is, the higher the expectation of an item, the smaller the amplitude. The amplitude variation of the positivity in the PSG condition, however, was most probably not caused by differences in expectancies. In the present study, the number of violations was counterbalanced over the positions in a sequence for both rule types. Due to this manipulation, the expectancy was held constant over the positions in a sequence. Moreover, if an increased expectation would be the basis of the reduced amplitude of the positivity at the fourth position in PSG, the same decrease of the amplitude would be expected in the FSG condition. This was not the case. Hence, differences in subjective probability can be excluded as a generator of the variation of the amplitude. Task relevance as a predictor of the varying amplitude can also be excluded. The attention to a task is directly proportional to the amplitude (where ignoring a task elicits no P300). In the present study, all stimuli were relevant to the task, and subjects reached a very high performance level (98\% correct answers in FSG, 96\% in PSG). Thus, 
task relevance could not have generated the amplitude differences of the late positivity in the PSG condition. However, another attribute of the P300 is the variability of the amplitude as a function of the conditional probability of an event; that is, the less expected the stimulus the larger the amplitude (Squires et al., 1976). Applying this to the present study, one could assume that the occurrence probability of a Category B item at the fourth position (incorrect item for long sequences and correct item for short sequences) is higher than a Category A item at the seventh position (only incorrect long sequence). An A at the end of the sequence is less likely than a $\mathrm{B}$ at the fourth position and the amplitude of the positivity is higher at the end of the sequence than at the fourth position of the sequence. This could also have had an influence on the modulation of the positivity amplitude.

The data at hand are in agreement with those reported by Nunez-Pena and Honrubia-Serrano (2004), Coulson et al. (1998), and Patel et al. (1998), who showed that the late positivity varied as a function of the saliency of the violation. Nunez-Pena and Honrubia-Serrano reported that the positivity elicited by the processing of numerical series varied as a function of the violation type; that is, the more salient the error, the higher the amplitude. In a study with anomalies in music, Patel et al. showed that the more distant a violating tone in a harmonic sequence from the expected tone, the higher the amplitude of the late positivity. Last, Coulson et al. reported that pronoun case violations in a sentence caused a higher amplitude of the late positivity than verb argument violations; that is, the more salient the morphosyntactic violation, the higher the amplitude of the positivity. In line with these findings, the results of the present study support the hypothesis that the late positivity reflects difficulty of integration, and, as will be discussed below, the degree of integration difficulty affects the size of the effect.

\section{Effect of Sequence Length: Long versus Short}

In the present study, short and long sequences were used in both rule types FSG and PSG. The manipulation of the sequence length caused a temporary ambiguity at the beginning of the sequence in PSG, but not in FSG; that is, a Category A syllable at the third position could be processed as a correct long sequence (AAAABBBB) or an incorrect short sequence (AA-AB), and vice versa for Category B. Post hoc analyses showed that subjects always treated those positions as members of long sequences. Moreover, the detection of an error took place at the item that immediately followed the violation at the third position, as revealed by a positivity at 950 to $1250 \mathrm{msec}$ after the onset of the third position. This was the case for both short and long sequences.

Two differences were observed in the ERP pattern between short and long sequences for the detection of a violation at the ambiguous third position. First, a second positivity was displayed at about 1450 to $1850 \mathrm{msec}$ after onset of the third position for short sequences only. As this component showed up at the end of the short sequence, this positivity could represent a confirmation effect of the error detection. Second, the effect of violation detection for long sequences $\left(\omega^{2}=.44\right)$ is higher than for short sequences $\left(\omega^{2}=.11\right)$. This result might suggest a difficulty of integration effect. Because it was shown that the third position was processed as if it were a long sequence, a $\mathrm{B}$ at the third position (incorrect/long) is more difficult to integrate than an A (incorrect/short) at this position. Hence, the greater amplitude of the error detection positivity for long sequences could have been caused by increased difficulty with integration in long sequences at the ambiguous third position.

\section{General Discussion}

The size of the violation effect systematically changes over error positions in the PSG, but not in the FSG. In PSG, the violation effect at the fourth position $\left(\omega^{2}=.17\right)$ was smaller than the effect of violation at later positions (sixth position, $\omega^{2}=.67$; seventh position, $\omega^{2}=.72$ ). At the early positions of the sequences, an structural violation is not as salient as it is at later positions. Because of the ambiguity at the beginning of a sequence, inserting a $\mathrm{B}$ at the beginning of the structure is not an error which is as salient as placing an A at the end of the structure. Hence, the integration of a non-expected syllable at the end of the structure is more difficult than at an earlier position. The difference observed for the two grammar types indicates that FSG and PSG are processed differently. The FSG can be processed on the basis of local rules (local probability), but the PSG cannot. Thus, the change in the amplitudes on the different violation positions in the PSG could reflect the hierarchical processing of the sequences. Still, this is a necessary, but not sufficient, argument for the hierarchical processing of the $\mathrm{A}^{n} \mathrm{~B}^{n}$ structure in the present experiment.

Although the late positivity in PSG could reflect partly overlapping neurophysiological generators with those used for natural language processing, the extent to which the $\mathrm{A}^{n} \mathrm{~B}^{n}$ structure can be taken to represent human language remains open. Natural grammars contain more complex syntactic rules than the rules used in the present artificial grammar design. $\mathrm{A}^{n} \mathrm{~B}^{n}$ sequences feature center-embedded syllables, comparable with human languages. The processing principles for these two types of center-embedded structures should be the same in the $\mathrm{A}^{n} \mathrm{~B}^{n}$ structure and in embedded sentences and should therefore recruit similar brain structures. Brain imaging studies suggest that Broca's area is involved when nonlocal dependency relations are to be processed both in natural languages (Ben-Shachar, Hendler, Kahn, Ben-Bashat, \& Grodzinsky, 2003; Röder, 
Stock, Neville, Bien, \& Rosler, 2002) and in language-like artificial grammars (Opitz \& Friederici, 2003), but that the frontal operculum and the adjacent ventral premotor cortex is activated when local syntactic probabilities are to be processed (Friederici, Ruschemeyer, Hahne, \& Fiebach, 2003; Friederici, Bahlmann, Heim, Schubotz, \& Anwander, 2006). This points towards a functional neuroanatomical separation, reflecting two different grammar types, rather than two different domains, that is, natural and artificial.

However, in the ERP the processing of syntactic violations is usually correlated with the ELAN effect. ${ }^{1}$ The ELAN component has been interpreted to reflect automatic aspects of local structure building during language processing (Hahne \& Friederici, 1999; Neville et al., 1993). Hoen and Dominey (2000) even assume that the LAN reflects structure-building processes in general. Further research is needed to explore whether the processing of the $\mathrm{A}^{n} \mathrm{~B}^{n}$ structure shares the early, automatic, violation detection mechanisms reflected by the ELAN in natural language processing.

\section{Summary}

Different ERP components were found for the processing of sequences of different structural types, namely, sequences characterized by transitional probabilities (FSG) and sequences characterized by hierarchical dependencies (PSG). The processing of the FSG elicited a posteriorly distributed early negativity and a late positivity in response to a local structural violation, independent of its position in the sequence. In contrast, the PSG revealed a positivity that varied as a function of the violation position within a hierarchically structured sequence. Violations introduced late in a PSG sequence elicited a higher amplitude of the positivity than violations at the beginning of a sequence. The positivity was therefore interpreted to reflect difficulty of integration. In contrast, the early negativity observed in the FSG seems to reflect an unfulfilled expectation of an upcoming category in a local transition.

\section{Acknowledgments}

The authors thank Kerrie Elston-Güttler, Korinna Eckstein, and Henning Holle for the fruitful discussions on the topic and Cornelia Schmidt for her help in data acquisition.

Reprint requests should be sent to Jörg Bahlmann, Max Planck Institute for Human Cognitive and Brain Sciences, PO Box 500 355, 04103 Leipzig, Germany, or via e-mail: bahlmann@cbs. mpg.de.

\section{Note}

1. As described in the Introduction, a LAN was sometimes associated with memory processes (Rösler et al., 1998; Kluender \& Kutas, 1993). In the present study, no LAN was found. Moreover, the higher amplitude of the positivity for later viola- tions could not be explained as an increase in memory load at the end of a sequence, as no changes in the amplitude of the positivity were observed between early and late violations in FSG, but only in PSG. Therefore, memory load could be excluded as a predictor of the different amplitudes of the positivity.

\section{REFERENCES}

Baldwin, K. B., \& Kutas, M. (1997). An ERP analysis of implicit structured sequence learning. Psychophysiology, 34, 74-86.

Ben-Shachar, M., Hendler, T., Kahn, I., Ben-Bashat, D., \& Grodzinsky, Y. (2003). The neural reality of syntactic transformations: Evidence from functional magnetic resonance imaging. Psychological Science, 14, 433-440.

Chomsky, N. (1965). Aspects of the theory of syntax. Oxford, England: MIT Press.

Chomsky, N., \& Miller, G. A. (1958). Finite state languages. Information \& Control, 1, 91-112.

Coulson, S., King, J. W., \& Kutas, M. (1998). ERPs and domain specificity: Beating a straw horse. Language $\mathcal{E}$ Cognitive Processes, 13, 653-672.

Dominey, P. F., Hoen, M., Blanc, J. M., \& Lelekov-Boissard, T. (2003). Neurological basis of language and sequential cognition: Evidence from simulation, aphasia, and ERP studies. Brain \& Language, 86, 207-225.

Donchin, E., \& Coles, M. G. (1988). Is the P300 component a manifestation of context updating? Behavioral and Brain Sciences, 11, 357-427.

Eimer, M., Goschke, T., Schlaghecken, F., \& Sturmer, B. (1996). Explicit and implicit learning of event sequences: Evidence from event-related brain potentials. Journal of Experimental Psychology: Learning, Memory, and Cognition, 22, 970-987.

Fiebach, C. J., Schlesewsky, M., \& Friederici, A. D. (2001). Syntactic working memory and the establishment of filler-gap dependencies: Insights from ERPs and fMRI. Journal of Psycholinguistic Research, 30, 321-338.

Fiebach, C. J., Schlesewsky, M., \& Friederici, A. D. (2002). Separating syntactic memory costs and syntactic integration costs during parsing: The processing of German WH-questions. Journal of Memory \& Language, 47, 250-272.

Fitch, W. T., \& Hauser, M. D. (2004). Computational constraints on syntactic processing in a nonhuman primate. Science, 303, 377-380.

Friederici, A. D., Bahlmann, J., Heim, S., Schubotz, R. I., \& Anwander, A. (2006). The brain differentiates human and non-human grammars: Functional localization and structural connectivity. Proceedings of the National Academy of Sciences of the United States of America, 103, 2458-2463.

Friederici, A. D., Pfeifer, E., \& Hahne, A. (1993). Event-related brain potentials during natural speech processing: Effects of semantic, morphological and syntactic violations. Cognitive Brain Research, 1, 183-192.

Friederici, A. D., Ruschemeyer, S. A., Hahne, A., \& Fiebach, C. J. (2003). The role of left inferior frontal and superior temporal cortex in sentence comprehension: Localizing syntactic and semantic processes. Cerebral Cortex, 13, 170-177.

Friederici, A. D., Steinhauer, K., \& Pfeifer, E. (2002). Brain signatures of artificial language processing: Evidence challenging the critical period hypothesis Proceedings of the National Academy of Sciences, U.S.A., 99, 529-534. 
Goschke, T., Friederici, A. D., Kotz, S. A., \& van Kampen, A. (2001). Procedural learning in Broca's aphasia: Dissociation between the implicit acquisition of spatio-motor and phoneme sequences. Journal of Cognitive Neuroscience, 13, 370-388.

Greenhouse S.W., \& Geisser, S. (1959). On methods in the analysis of profile data. Psychometrica, 24, 95-112.

Gunter, T. C., Friederici, A. D., \& Schriefers, H. (2000). Syntactic gender and semantic expectancy: ERPs reveal early autonomy and late interaction. Journal of Cognitive Neuroscience, 12, 556-568.

Gunter, T. C., Stowe, L. A., \& Mulder, G. (1997). When syntax meets semantics. Psychophysiology, 34, 660-676.

Hahne, A., \& Friederici, A. D. (1999). Electrophysiological evidence for two steps in syntactic analysis. Early automatic and late controlled processes. Journal of Cognitive Neuroscience, 11, 194-205.

Hahne, A., \& Friederici, A. D. (2002). Differential task effects on semantic and syntactic processes as revealed by ERPs. Cognitive Brain Research, 13, 339-356.

Hays, W. L. (1973). Statistics for the social sciences (2nd ed.). New York: Holt, Rinehart and Winston.

Hoen, M., \& Dominey, P. F. (2000). ERP analysis of cognitive sequencing: A left anterior negativity related to structural transformation processing. NeuroReport, 11, 3187-3191.

Johnson, R., Jr., \& Donchin, E. (1982). Sequential expectancies and decision making in a changing environment: An electrophysiological approach. Psychophysiology, 19, 183-200.

Kaan, E., Harris, A., Gibson, E., \& Holcomb, P. (2000). The $\mathrm{P} 600$ as an index of syntactic integration difficulty. Language \& Cognitive Processes, 15, 159-201.

King, J. W., \& Just, M. A. (1991). Individual differences in syntactic processing: The role of working memory. Journal of Memory \& Language, 30, 580-602.

King, J. W., \& Kutas, M. (1995). Who did what and when? Using word- and clause-level ERPs to monitor working memory usage in reading. Journal of Cognitive Neuroscience, 7, 376-395.

Kluender, R., \& Kutas, M. (1993). Bridging the gap: Evidence from ERPs on the processing of unbounded dependencies. Journal of Cognitive Neuroscience, 5, 196-214.

Lelekov-Boissard, T., \& Dominey, P. F. (2002). Human brain potentials reveal similar processing of non-linguistic abstract structure and linguistic syntactic structure. Clinical Neurophysiology, 32, 72-84.

Münte, T. F., Heinze, H. J., Matzke, M., Wieringa, B. M., \& Johannes, S. (1998). Brain potentials and syntactic violations revisited: No evidence for specificity of the syntactic positive shift. Neuropsychologia, 36, 217-226.

Neville, H. J., Nicol, J. L., Barss, A., Forster, K. I., \& Garrett, M. (1991). Syntactically based sentence processing classes: Evidence from event-related brain potentials. Journal of Cognitive Neuroscience, 3, 151-165.

Nissen, M. J., \& Bullemer, P. (1987). Attentional requirements of learning: Evidence from performance measures. Cognitive Psychology, 19, 1-32.

Nunez-Pena, M. I., \& Honrubia-Serrano, M. L. (2004). P600 related to rule violation in an arithmetic task. Cognitive Brain Research, 18, 130-141.

Oken, B. S., \& Chiappa, K. H. (1986). Statistical issues concerning computerized analysis of brainwave topography. Annals of Neurology, 19, 493-497.

Opitz, B., \& Friederici, A. D. (2003). Interactions of the hippocampal system and the prefrontal cortex in learning language-like rules. Neuroimage, 19, 1730-1737.

Osterhout, L., \& Nicol, J. (1999). On the distinctiveness, independence, and time course of the brain responses to syntactic and semantic anomalies. Language \& Cognitive Processes, 14, 283-317.

Patel, A. D., Gibson, E., Ratner, J., Besson, M., \& Holcomb, P. J. (1998). Processing syntactic relations in language and music: An event-related potential study. Journal of Cognitive Neuroscience, 10, 717-733.

Röder, B., Stock, O., Neville, H., Bien, S., \& Rosler, F. (2002). Brain activation modulated by the comprehension of normal and pseudo-word sentences of different processing demands: A functional magnetic resonance imaging study. Neuroimage, 15, 1003-1014.

Rösler, F., Pechmann, T., Streb, J., Röder, B., \& Hennighausen, E. (1998). Parsing of sentences in a language with varying word order: Word-by-word variations of processing demands are revealed by event-related brain potentials. Journal of Memory \& Language, 38, 150-176.

Rüsseler, J., Hennighausen, E., Münte, T. F., \& Rösler, F. (2003). Differences in incidental and intentional learning of sensorimotor sequences as revealed by event-related brain potentials. Cognitive Brain Research, 15, 116-126.

Rüsseler, J., \& Rösler, F. (2000). Implicit and explicit learning of event sequences: Evidence for distinct coding of perceptual and motor representations. Acta Psychologica, 104, 45-67.

Squires, K. C., Wickens, C., Squires, N. K., \& Donchin, E. (1976). The effect of stimulus sequence on the waveform of the cortical event-related potential. Science, 193, $1142-1146$. 